Illinois State University

ISU ReD: Research and eData

Theses and Dissertations

$4-12-2014$

\title{
The Effects of a Foot-Toe Orthosis on Dynamic Balance and Hallux Valgus Angle
}

Adam Richard Kelly

Illinois State University, arkelly@ilstu.edu

Follow this and additional works at: https://ir.library.illinoisstate.edu/etd

Part of the Biomechanics Commons, and the Physical Therapy Commons

\section{Recommended Citation}

Kelly, Adam Richard, "The Effects of a Foot-Toe Orthosis on Dynamic Balance and Hallux Valgus Angle" (2014). Theses and Dissertations. 161.

https://ir.library.illinoisstate.edu/etd/161

This Thesis is brought to you for free and open access by ISU ReD: Research and eData. It has been accepted for inclusion in Theses and Dissertations by an authorized administrator of ISU ReD: Research and eData. For more information, please contact ISUReD@ilstu.edu. 


\title{
THE EFFECTS OF A FOOT-TOE ORTHOSIS ON DYNAMIC BALANCE AND
}

\section{HALLUX VALGUS ANGLE}

\author{
Adam R. Kelly
}

37 Pages

May 2014

Context: Decreased balance has been identified as a risk factor for lower extremity injury in several populations. Previous literature has investigated the effects of using orthotics, textured insoles and textured surfaces on balance. However, no research exists on the effects of a foot-toe orthosis on dynamic balance. Objective: To determine the effects of a foot-toe orthosis on dynamic balance and hallux valgus angle. Design: A randomized controlled trial. Setting: Athletic Training research laboratory. Participants: Sixty-three healthy and recreationally active collegiate students. (age: $21.59 \pm 1.49 y$ rs, height $172.48 \pm 8.99 \mathrm{~cm}$, mass $73.86 \mathrm{~kg} \pm 15.64 \mathrm{~kg}$ ) Interventions: Participants were randomly allocated to one of three groups that received either the foot-toe orthosis and control shoe (FTO), the control shoe only (SO), or a true control group (CO). Main Outcome Measures: The composite score for the Star Excursion Balance Test (SEBT) was used to quantify dynamic balance at baseline and follow-up. The hallux valgus angle was assessed using a goniometer. Results: Hallux valgus angle did not change significantly $(p=.380, p=.298)$ over the 4-week time period for either FTO or SO groups. Significant improvements in dynamic balance were found in the FTO group $(p=.001)$ and SO group ( $p=.026)$ at the 4-week follow-up compared with the CON group. Conclusion: 
Four weeks of intervention with a foot-toe orthosis and the control shoe may significantly improve dynamic balance in a young and healthy collegiate population. Key Words: Proprioception, Ankle, Foot, Range of Motion, Y Balance Test, Bunion, Falls, Somatosensory 
THE EFFECTS OF A FOOT-TOE ORTHOSIS ON DYNAMIC BALANCE AND

HALLUX VALGUS ANGLE

ADAM R. KELLY

A Thesis Submitted in Partial

Fulfillment of the Requirements

for the Degree of

MASTER OF SCIENCE

School of Kinesiology and Recreation

ILLINOIS STATE UNIVERSITY

2014 
THE EFFECTS OF A FOOT-TOE ORTHOSIS ON DYNAMIC BALANCE AND

HALLUX VALGUS ANGLE

ADAM R. KELLY

COMMITTEE MEMBERS:

Justin M. Stanek, Chair

Michael R. Torry 


\section{ACKNOWLEDGMENTS}

I would like to thank my committee, Drs. Justin Stanek and Michael Torry, who guided me through the entire process. Thank you to Dr. Noelle Selkow for both her input and advice. A thank you goes out to both Correct Toes and Lems Shoes for donating their products for this study. I would also like to thank Kevin Morris for all of his assistance with data collection. Finally, I would like to thank my significant other, Nicole, for putting up with me through this entire process.

A. K. 


\section{CONTENTS}

\section{Page}

ACKNOWLEDGEMENTS $\quad$ i

CONTENTS

TABLES

FIGURES $\quad$ iv

CHAPTER

I. INTRODUCTION: PURPOSE OF RESEARCH 1

II. REVIEW OF RELATED LITERATURE 4

Introduction 4

Bony Anatomy 4

Capsuloligamentous Structures $\quad 7$

Balance 11

Mechanoreceptors $\quad 14$

Plantar Pressure Stimulation and Orthotic Interventions $\quad 16$

$\begin{array}{ll}\text { III. METHODS } & 19\end{array}$

$\begin{array}{ll}\text { Participants } & 19\end{array}$

Instrumentation $\quad 20$

Measurements 22

Procedures $\quad 24$

Statistical Analysis $\quad 26$

$\begin{array}{ll}\text { IV. RESULTS } & 27\end{array}$

V. DISCUSSION AND CONCLUSION 29

Discussion $\quad 29$ 
REFERENCES 


\section{TABLES}

Table $\quad$ Page

1. Participant Characteristics by Group 20

2. Descriptive Statistics for YBT-LQ 28 


\section{FIGURES}

Figure $\quad$ Page

1. Y Balance Kit 21

2. Correct Toes 21

3. Control Shoe 21

4. Pre vs. Post YBT-LQ Composite Scores Chart 28 


\section{CHAPTER I}

\section{INTRODUCTION: PURPOSE OF RESEARCH}

Over $50 \%$ of all athletic injuries occur in the lower extremity according to the National Collegiate Athletic Association (NCAA) Injury Surveillance System injury data from 1998 to $2004 .{ }^{1}$ It has been reported that lateral ankle sprains are the most commonly occurring injury in physically active people ${ }^{2}$ and it is estimated that more than 23,000 people in the United States suffer an ankle sprain every day. ${ }^{3}$ Several risk factors for athletic injury have been identified in the literature and include history of previous injury, being female, anatomical mal-alignments, decreased muscle flexibility, decreased vertical jump height, tape or brace use and poor balance. ${ }^{4}$ While some of these factors may be non-modifiable, others, such as dynamic balance, can be readily evaluated and enhanced in the lab and clinical settings. It has also been reported that decreased balance can be found in individuals that have suffered ankle injuries and may be a significant risk factor for re-injury. In addition, decreased balance has also been identified as a risk factor for acute injury in healthy subjects. ${ }^{2}$

Dynamic balance is defined as a person's ability to maintain stability of the center of gravity (COG) within the base of support (BOS) while allowing desired movements., ${ }^{4,5}$ This is an important and complex component of human motor function, and is the product of information processed by the central nervous system (CNS) received from the visual, vestibular, dental occlusion and somatosensory systems using both feed-forward and feedback loops. ${ }^{6-12}$ However, the CNS tends to rely heavily upon only one system at 
a time. In healthy adults under normal conditions, the preferred input for balance and stability is that provided by the somatosensory or proprioceptive systems. ${ }^{13}$

The proprioceptive system is influenced by a continuous afferent feedback loop from mechanoreceptors in the skin, muscles, tendons, and joints. ${ }^{14}$ The mechanoreceptors process the stimuli of touch, pressure, vibration, tickle, and sense of position. ${ }^{7}$ This continuous feedback (combined with information from the vestibular and visual systems) provides for constant adjustments by the neuromuscular system to maintain equilibrium and stability. ${ }^{6}$ Adequate levels of balance are needed during both static and dynamic activities to successfully execute motor tasks and to prevent falls.

The Star Excursion Balance Test (SEBT) has been utilized in the literature as a reliable and efficient way to assess dynamic balance. ${ }^{15,16}$ The SEBT is a dynamic test that requires strength, flexibility, and proprioception. ${ }^{15}$ The SEBT tasks an individual with maintaining single leg stance on one leg while reaching as far as possible with the contralateral leg before returning to a double leg stance at the start position while under control. Several authors have found that the SEBT was able to predict injury risk in several populations such as collegiate football players, high school basketball players, and physically active college students. ${ }^{4,17,18}$ The SEBT is potentially a reliable and fieldexpedient way for clinicians and researchers to screen, assess, and treat the dynamic balance of individuals and thus monitor and/or modify their risk of lower extremity injury.

It has previously been investigated that dynamic balance may be influenced with orthoses and insoles through various theorized models of efficacy. ${ }^{14,19-32}$ The use of foot orthotics, textured insoles and textured surfaces to alter dynamic balance is not a novel 
approach. ${ }^{14,20,22,23,27,30,31,33-38}$ Nevertheless, there has been no studies to date on foot-toe orthoses and their effects on dynamic balance. Correct Toes ${ }^{\circledR}$ is a foot-toe orthosis and is designed to fit between the $1^{\text {st }}$ and $5^{\text {th }}$ phalanges of the foot. To date, there have been no studies investigating the effects of foot-toe orthoses on dynamic balance. Therefore, the purpose of this study was to determine the effects of the foot-toe orthosis on dynamic balance and hallux valgus angle. 


\section{CHAPTER II}

\section{REVIEW OF RELATED LITERATURE \\ Introduction}

\section{Bony Anatomy}

The lower leg is composed of two bones: medially is the thick and strong tibia and laterally, is the thin and slender fibula. These two bones are connected along their shafts by an interosseous membrane. The tibia is the primary weight-bearing bone of the crural region. Superiorly, the tibia has two flat articular surfaces known as the medial and lateral condyles. A small ridge known as the intercondylar eminence separates the condyles. This is the site of the tibiofemoral joint and is where the tibia articulates with the femur. Just distal to the condyles on the anterior surface of the tibia is a rough surface known as the tibial tuberosity and this is the insertion point for the thigh muscles responsible for extending the knee. The distal end of the tibia is relatively flat and is articulation with the talus. Medially is a large projection known as the medial malleolus, where there are many ligamentous insertion points that provide static restraints to ankle motion. This projection is easily palpable and visible through the skin. Moving laterally on the surface tibia is the fibular notch. This is the distal location of articulation between the tibia and fibular and forms the distal tibiofibular joint. ${ }^{39}$

The fibula is the slender and lateral bone that assists in stabilizing the ankle. The fibula is not a true weight-bearing bone. Proximally, the fibula is thicker and broader at the head, which is known as the apex at the most superior aspect of the fibular head. The 
distal end of the fibula much like the tibia has a large projection that is easily palpable and visible with the naked eye. This distal most aspect of the fibula is known as the lateral malleolus of the ankle. ${ }^{39}$

The next portion of the lower leg is the foot. The foot is composed of the tarsal bones, metatarsal and phalange bones. The tarsal bones consist of seven bones that are arranged in proximal and distal rows much like the carpal bones of the wrist. The shape and setup of the tarsal bones is drastically different due to the weight-bearing nature of these bones. The first bone of the proximal row and the largest tarsal bone is the calcaneus. The posterior surface of the calcaneus has the insertion point for the calcaneal or Achilles tendon. Superior to the calcaneus is the talus, which is the second largest tarsal bone. The talus has three different articulations: there is an inferoposterior articulation with the calcaneus, a superior trochlear surface where it articulates with the tibia, and an anterior area of translation with the navicular bone. The navicular bone is the last bone of the proximal row and is described as a short but wide tarsal bone on the medial aspect of the foot. The navicular helps to transfer motion between the rearfoot and forefoot segments. ${ }^{40}$ The distal row of the tarsal bones is composed of four bones. Listed with respect from medial to lateral locations this row contains the medial, intermediate and lateral cuneiforms as well as the cuboid bone. The cuboid bone is the largest bone of this group. ${ }^{39}$

Moving distally, the next section of the foot contains the metatarsal bones. The metatarsals are composed of five long bones and they are numbered I to V with respect from medial to lateral locations. Metatarsals I-III articulate at their base with the medial, intermediate and lateral cuneiform bones, respectively. Metatarsals IV and V articulate 
with the cuboid bone. Unlike the other metatarsal bones, metatarsal I has two sesamoid bones located at the distal plantar aspect or the head of the metatarsal. The sesamoid bones are analogous to an anatomical pulley system for the flexor hallucis brevis muscle. It also works to protect the flexor hallucis longus muscle tendon from trauma due to the weight-bearing role of the hallux..$^{39,40}$

The final portion of the foot and most distal aspect is composed of the phalanges. Starting medial lies the hallux or "great toe" and it is composed of only two bones, the proximal and distal phalanxes. Phalanges II-V are all composed of three separate phalanx bones. The hallux is the primary weight-bearing phalange. The phalanges are all slightly concave on their ventral aspect and each consist of a base, body and head. ${ }^{39}$

Traditionally, the bones of the foot have been split into three distinct areas known as the forefoot, midfoot, and the rearfoot. The forefoot consists of the five metatarsals and 14 phalanges. The midfoot is composed of the navicular, cuboid, and all three cuneiform bones. Finally, the rearfoot or hindfoot as it is also known encompasses the calcaneus and the talus. ${ }^{40}$ These three major segments of the foot work in conjunction to form three distinct arches of the foot. There are two longitudinal arches, the medial and lateral as well as the transverse arch. These arches work to support the foot over various terrain while transferring bodyweight and forces throughout the kinetic chain. They are not solid arches but instead are "springy" to help absorb and store energy with walking and running. 39,40

The medial longitudinal arch stretches from the anteroinferior calcaneus to the hallux and consists of the calcaneus, talus, navicular, cuneiforms, and metatarsals I through III. The lateral longitudinal arch also begins at the anteroinferior calcaneus and 
reaches to metatarsal V. It is composed of the calcaneus, cuboid, cuneiforms as well as metatarsals IV and V. ${ }^{39}$ Primary support for the longitudinal arches comes from the plantar calcaneonavicular or "spring" ligament, long plantar ligament, plantar fascia or plantar aponeurosis and the plantar calcaneocuboid ligament. The longitudinal arches also find dynamic support from muscle tension of the lower leg, especially that provided by the tibialis posterior muscle. ${ }^{40}$

The transverse arch includes the cuboid, cuneiforms and proximal heads of the metatarsal bones. Support of the transverse arch comes from the transverse head of the adductor hallucis muscle and the intermetatarsal ligaments. ${ }^{39}$

\section{Capsuloligamentous Structures}

The tibia and fibula articulate at proximal and distal locations. The proximal tibiofibular articulation is a plantar synovial joint that is supported by strong anterior and posterior ligaments. The distal tibiofibular articulation, however, is a syndesmosis or a fibrous connective tissue that connects the two bones together. The tibiofibular articulations do not have a joint capsule but the anterior and posterior tibiofibular ligaments do provide stability. Additional stability is provided the crural interosseous ligament, which is part of the interosseous membrane. ${ }^{40}$

The talocrural joint is formed by the talus, tibia and lateral malleolus of the fibula. It is a uniaxial, modified synovial hinge joint. The distal end of the tibia is concave and articulates with the convex superior aspect of the talus and this creates the medial border and roof of the ankle mortise. While the tibia is the main weight-bearing bone of the lower leg the fibula can support up to $17 \%$ of the load and serves as the lateral border of 
the ankle mortise. The fibula also serves as a site for muscular and ligamentous attachments. The closed pack position of the joint is maximum dorsiflexion because the talus is wider on the anterior aspect. ${ }^{40}$

Despite having a thin joint capsule the talocrural joint is supported by many strong ligaments. Medially, there are four bands of the medial collateral ligament or "deltoid" ligament for short. Anteriorly, there are the anterior tibiotalar and tibionavicular ligaments, which are taut when the subtalar joint is plantarflexed. The tibiocalcaneal and posterior tibiotalar ligaments are taught with dorsiflexion and are located posteriorly.

The lateral aspect of the ankle has three main supporting ligaments. The anterior talofibular ligament resists inversion during plantar flexion and also resists anterior translation of the talus. The calcaneofibular ligament resists extreme dorsiflexion and is the primary restraint for talar inversion when the ankle is in its middle arc of available range of motion. The final and most posterior of the lateral restraints is the posterior talofibular ligament. This ligament is able the resist the most force of the three and resists posterior displacement of the talus. These three ligaments are relatively weak compared to the medial deltoid ligaments. This weakness combined with less overall bony stability on the lateral aspect results in a greater frequency of lateral ankle sprains. ${ }^{40}$

The subtalar joint is located at the articulation of the sustentaculum tali of the superior calcaneous and the inferior facets of the talus. The subtalar joint functions as an adaptable structure. Motion occurs only with stretching of small ligaments during weightbearing activities. This motion occurs with ovoid bone surfaces sliding atop reciprocally shaped ovoid bone surfaces. The closed packed position of the subtalar joint is vertical 
loading with internal rotation. The subtalar joint finds stability through ligamentous attachments through the tarsal canal. The tarsal canal is a sulcus that gives space for an intra-articular ligament. This ligament is known as the talocalcaneal interosseous ligament and it divides the joint into two articular cavities and serves as the axis for talar tilt. It also is provides joint stability, especially with supination. There are also four small talocalcaneal ligaments that connect between the two tarsal bones. ${ }^{40}$

The transverse tarsal joint is composed of two articulations known as the calcaneocuboid joint and the talonavicular joint. They are known together as the transverse tarsal joint due to their juxtaposed function and location. The calcaneocuboid joint is saddle-shaped and supination is its close-packed position. The calcaneocuboid joint is non-axial and has only limited gliding motion. Soft tissue support comes from the bifurcate, plantar and dorsal calcaneocuboid, and the long plantar ligaments. The talonavicular joint is a modified ball and socket joint and its close-packed position is in full supination. Movements that occur at this joint include gliding and rotation. Three primary restraints of the talonavicular joint are the plantar calcaneonavicular or spring ligament on the inferior aspect; the deltoid ligament on the medial aspect and the bifurcate ligament support the lateral aspect. The talus articulates with the navicular while simultaneously allowing motion on the calcaneus or subtalar joint. This combined movement results in a reference to the talonavicular joint as the talocalcaneonavicular joint. This is also because any motion occurring at the subtalar joint results in similar motion at the transverse tarsal joint. ${ }^{40}$

The tarsometatarsal and intermetatarsal joints are both supported by the deep transverse metatarsal ligament. Both of these joints are classified as gliding joints and 
have a close-packed position with supination. The architecture of these joints allows for the foot to adapt to a variety of terrain and uneven surfaces. ${ }^{40}$

The plantar fascia, also known as the plantar aponeurosis, is a thick band of fascia that spans the plantar surface of the foot. This thick band of soft tissue provides support for the longitudinal arches and has three divergent segments. The main portion of the plantar fascia reaches from the posteromedial calcaneal tubercle and inserts into the distal plantar aspects of the proximal phalanges of each toe. Here it attaches with the deep transverse metatarsal ligaments. The medial and lateral portions deviate from the central segment as it extends more distally down the foot. The plantar fascia can stretch between $9-12 \%$ of its resting length during the gait cycle to act as a "spring" to store mechanical energy. This energy can then be released to assist with toe-off and propel the body forward more efficiently. ${ }^{40}$

The metatarsophalangeal joint is classified as a condyloid joint and full extension is its close-packed position. The proximal and distal interphalangeal joints are hinge joints that also have full extension as a close-packed position. The metatarsophalangeal joints are supported and covered by a fibrous joint capsule and the plantar aspect is also supported by the plantar fascia and plantar ligament. The collateral ligaments also support the medial and lateral portions of the joint capsule. The proximal and distal interphalangeal joints find stability from the plantar and dorsal joint capsule as well as the collateral ligaments. ${ }^{40}$

The muscles, nerves, and blood vessels of the lower leg are separated into four compartments by thick casings of fascia. The four sections are known as the anterior, 
deep posterior, superficial posterior and lateral compartments. Inside the anterior compartment is the tibialis anterior, extensor digitorum longus, extensor hallucis longus, and peroneus tertius if it is present. The deep posterior compartment contains the tibialis posterior, flexor digitorum longus, flexor hallucis longus, the tibialis posterior artery, and the tibial nerve. The superficial posterior compartment includes the gastrocnemius, soleus, and plantaris muscles. Finally, the lateral compartment holds the peroneus longus and peroneus brevis muscles. ${ }^{40}$

Apart from the extrinsic muscles of the foot that cross the ankle and stem from the lower leg, the foot also contains many intrinsic muscles as well. The dorsal aspect of the foot contains only one intrinsic muscle and that is the extensor digitorum brevis. The most superficial layer of intrinsic muscles on the ventral or plantar aspect of the foot contains the flexor digitorum brevis, the abductor digiti minimi and the abductor hallucis muscles. The next deepest layer holds the quadratus plantae and the lumbricals of the foot. The third deepest layer contains the adductor hallucis, flexor digiti minimi brevis and the flexor hallucis brevis. Finally, the deepest layer of intrinsic muscles on the plantar aspect of the foot contains the dorsal interosseous muscles and the plantar interosseous muscles. ${ }^{39,40}$

\section{Balance}

Balance is the ability to maintain equilibrium or balance while standing upright. Specifically, a person's central nervous system (CNS) and neuromuscular system must work together to control their center of gravity (COG) within their base of support (BOS). ${ }^{7}$ This is a complex interaction involving the processing and coordination of 
sensory, motor and biomechanical components..$^{6-9}$ An individual perceives their position in space with relation to gravity and their surroundings by combining information from the visual, vestibular and somatosensory systems.

Postural stability is the function of a feedback control circuit between the CNS and musculoskeletal system. In response to this feedback loop, the musculoskeletal system produces reactive motions at the trunk, hip, knee, and ankle joints to maintain postural control. ${ }^{7,13,41}$ Medical conditions affecting mental status, nervous disorders, vestibular function, and fatigue can have detrimental effects to postural stability. ${ }^{42}$

The vestibular system is one of the three key systems used in the maintenance of postural stability. The vestibular apparatus is the sensory organ of the vestibular system and its role is to detect sensations for maintaining equilibrium and is located within the ear. Initially, the ear only functioned for the purpose of equilibrium in vertebrates and only later did the ear evolve the capacity for auditory function. The vestibular apparatus is located in a bony labyrinth located within the temporal bone and is comprised of tiny bony tubules and chambers. This labyrinth is located alongside the cochlear duct of the inner ear. It also contains three semicircular ducts as well as the anterior saccule and the posterior utricle, both of which are large bony chambers. ${ }^{7,39}$

Afferent feedback from the vestibular apparatus is processed for three distinct purposes. The information coordinates the musculature of the eye so that when movement of the head occurs, the eyes are able to remain fixated upon one object. This sensory information is also processed to maintain an upright posture. Finally, the information provided by the vestibular apparatus is used to provide a conscious 
awareness of body position and acceleration once processed by the thalamus and relayed to the cortex. ${ }^{7,39}$

Researchers ${ }^{13,43,44}$ consider signals from the vestibular system to be the primary input utilized by the CNS when stabilizing slow body sway. This is achieved with lowlevel activation of lower leg musculature in response to this input. During normal conditions the CNS relies primarily on input from the visual and somatosensory systems to maintain balance. However, input from the vestibular system is crucial in maintaining equilibrium when rapid changes or perturbations result in a change of head position or direction of movement. This input allows for an important automatic control mechanism that stabilizes the direction of gaze. ${ }^{7}$ More specifically, it allows for proper functioning of the vestibulo-ocular reflex. This reflex functions in response to signaling from the semicircular ducts by rotating the eyes in an equal and opposite direction of the head when it is tilted. This allows for proper processing of visual information in the maintenance of equilibrium and balance as well. ${ }^{7,39}$

The vestibular, visual and somatosensory systems all contribute input for maintaining postural stability. ${ }^{6-9}$ However, the central nervous system tends to rely heavily upon only one system at a time. In healthy adults under normal conditions, the preferred input for balance and stability is that provided by the somatosensory or proprioceptive system. ${ }^{13}$ The somatosensory system derives much of its input via a continuous afferent feedback loop from various types of mechanoreceptors that are located in the skin, muscles, tendons and joints. ${ }^{14}$ These mechanoreceptors process different stimuli such as touch, pressure, vibration, tickle and sense of position. ${ }^{7}$ This continuous feedback (along with information from the vestibular and visual systems) 
provides for constant adjustments by the neuromuscular system in the maintenance of equilibrium and postural stability. ${ }^{6,45,46}$

\section{Mechanoreceptors}

The human body contains several different types of mechanoreceptors that respond to signals such as vibration, touch, pressure, stretch or tension. This includes the organs of auditory function and balance and can be found in the skin, viscera and joints of the body. ${ }^{39}$ Mechanoreceptors are composed of relatively simple but varied nerve cells. Most mechanoreceptors can be classified by encapsulated nerve endings but some have unencapsulated nerve endings as well. Encapsulated nerve endings are nerve fibers wrapped in connective tissue or glial cells. Unencapsulated nerve endings are not wrapped in connective tissue and are sensory dendrites. ${ }^{39}$

An example of a mechanoreceptor with an unencapsulated nerve ending would be a Merkel disc or tactile disc. They are tonic receptors of light touch and are believed to sense texture, edges and shapes. These are flattened nerve endings associated with Merkel cells located at the base of the epidermis. ${ }^{39}$

There are many more mechanoreceptors with encapsulated nerve endings. One example would be Meissner or tactile corpuscles. These are phasic receptors that detect light touch and texture. These cells occur in the dermal papillae of the skin but are limited to sensitive hairless areas of the body. Meissner corpuscles are what allow a person to

detect the difference between sensations like silk and sandpaper. ${ }^{39}$

Another example of this class of mechanoreceptor would be lamellated or pacinian corpuscles. They are also phasic receptors that sense deep pressure, stretch, 
tickle, and vibration. These cells are located in the periosteum of bone, joint capsules, pancrease and other various viscera. They are also found deep in the dermis such as the hands, feet, breasts and genitals. ${ }^{39}$

Ruffini corpuscles are another type of mechanoreceptors with encapsulated nerve endings. They are tonic receptors that sense heavy touch, pressure, stretching of the skin and joint movement. These nerve endings are located in the dermis, subcutaneous tissue, ligaments, tendons and joint capsules. ${ }^{39}$

Finally, the Golgi tendon organs (GTO) and the muscle spindles are two mechanoreceptors that are of major importance to the neuromuscular system. They are both classified as encapsulated nerve endings. GTOs are proprioceptors located in a tendon near the musculotendinous junction. When the tendon is slack, the collagen fibers are spread out and place minimal pressure on the GTO nerve endings spread amongst them. When a muscle contraction puts tension on the tendon, the collagen fibers squeeze together and compress the nerve endings between them. These afferent nerve fibers then relay information to the spinal cord and the CNS that provides feedback on the amount of muscle tension about a joint.

The muscle spindles are proprioceptive cells with a fusiform shape. They are dispersed throughout the muscle belly and they run parallel with muscle fibers. They are concentrated near the musculotendinous junction. Muscle spindles are composed of 3-12 modified muscle fibers and a few nerve fibers and a fibrous capsule surrounds them and blends the spindle into the endomysium of the muscle. The modified muscle fibers located within the spindle are known as intrafusal muscle fibers and the fibers outside of 
the spindle are known as extrafusal muscle fibers. ${ }^{39}$ Muscle spindles process and relay information to the nervous system regarding both muscle length and the rate of change in length. A muscle spindle splits into different afferent pathways as it enters the central nervous system. One of these pathways can stimulate efferent motor neurons if the muscle spindle senses a muscle being stretched. This reflex arc is important for maintaining equilibrium and posture and is known as the stretch reflex or myotatic reflex. ${ }^{7,39}$

\section{Plantar Pressure Stimulation and Orthotic Interventions}

Stimulating the mechanoreceptors of the foot to investigate the effects on balance and postural stability is not a novel concept in the research literature and while there are conflicting results there is evidence to show that these devices may help to increase postural stability. Previous research has looked at the effects of using various modalities such as orthoses, textured insoles, and textured surfaces to stimulate the plantar cutaneous and mechanoreceptors of the foot and ultimately affect balance. .4,20,22,23,27,30,31,33-38 $^{1}$

Gross et al. ${ }^{22}$ found a significant improvement in selected measures of balance immediately and 2 weeks after a custom foot orthoses intervention in a group of elderly adults volunteers, a population of subjects that is at risk for poor balance and falls.

Hamlyn et al. ${ }^{38}$ found that prefabricated orthotic intervention lead to improvements in postural stability in a population of subjects with functional ankle instability. The use of orthotics has also been found to decrease postural sway in injured subjects after suffering an acute inversion ankle sprain. ${ }^{23}$ Some researchers postulate that poor foot posture or structural mal-alignment may be a factor in decreased postural stability. ${ }^{19,47}$ Interestingly, 
Cobb et al. ${ }^{20}$ and Mattacolla et al ${ }^{26}$ found that a 6 -week orthotic intervention in a population of seemingly healthy subjects but with poor foot alignment was able to significantly increase postural stability. These results were inconsistent with findings from previous research ${ }^{25,28}$ and it is suggested that the differences were an artifact of differences in orthotic construction, definition of foot mal-alignment, and measures of postural stability.

Textured Insoles are a quick and cost effective method of attempting to increase postural stability. The purpose of textured insoles is to increase afferent proprioceptive feedback and consequently increase postural stability. Corbin et al. ${ }^{14}$ found that by inserting a textured insole into the footwear of a population of healthy subjects they were able to able to increase postural stability in bilateral stance but found no significant change for unilateral stance. Conversely, McKeon et al. ${ }^{27}$ found that postural stability decreased in a population of subjects with chronic ankle instability when utilizing textured insoles with footwear. However, these authors noted that this provided evidence that afferent stimulation did have an effect on postural control despite being negative in this injured population. Other investigators ${ }^{30,31,33,36}$ found that textured insoles were efficacious for increasing the postural stability in an elderly but relatively healthy population. Additionally, Palleul et al. ${ }^{30}$ found the textured insoles in their study to be efficacious for a population of healthy young adults as well. Interestingly, it was noted in the study by Perry et al. ${ }^{31}$ that the insoles were still effective even after 12 weeks of continuous use. This provides support for the idea that the sensory motor system requires constant afferent feedback for controlling postural stability and potentially represents a long-term solution for individuals with postural stability impairments. 
Recently, it has been suggested ${ }^{35,37,48,49}$ that even just standing on textured surfaces is enough to increase postural stability. Matsusaka et al. ${ }^{49}$ even found that the application of tape to the plantar aspect of the foot was a significant enough proprioceptive input to increase postural stability more quickly than a control group during a balance training program utilizing a balancing disk surface. Additionally, Hoffman et al. ${ }^{48}$ found that a textured balancing disk was able to increase postural stability with a 10 week training program but this study suffered from a lack of control group that utilized a non-textured balancing disk. Futhermore, Hatton et al. ${ }^{35,37}$ found evidence in two separate studies that standing on a textured surface could increase postural stability in a healthy elderly population. There was also evidence to support the idea that different textures could have different effects on postural stability and that some textures may be beneficial while other surfaces not. ${ }^{35}$ 


\section{CHAPTER III}

\section{METHODS}

This study was a controlled laboratory study with randomization that investigated the effects of a foot-toe orthosis and control shoe on dynamic balance and hallux valgus angle. The independent variable was group (Foot-toe orthosis and control shoe, control shoe only, or true control group). The dependent variables were dynamic balance and hallux valgus angle.

\section{Participants}

Sixty-three healthy, and physically active college students between the ages of 18 and 29 years (See Table 1.) volunteered to participate in the study. All of the participants provided written informed consent before participation, and the university's institutional review board approved the study. Participants were randomly allocated into one of three groups by using an online random group allocation generator (http://www.graphpad.com/quickcalcs/index.cfm). The intervention ( $\mathrm{n}=24)$ group consisted of subjects receiving both the foot-toe orthoses and the control shoes. The shoeonly group $(n=21)$ consisted of subjects receiving the control shoe but not the orthoses, and there was also a true control group $(n=18)$ where subjects did not receive any intervention. Participants were eligible to participate if they were physically active for at least 30 minutes a day, 3 times a week or 90 minutes total per week. Volunteers were ineligible if they had a history of lower extremity injury, concussion or pathology that 
could affect equilibrium or neuromuscular control within the past six months or any history of lower extremity surgery. Leg dominance was operationally defined as the preferred leg that a subject would use to kick a ball with and was determined using a preparticipation questionnaire that was filled upon arrival along with other demographic inquiries.

Table 1. Participant Characteristics by Group

\begin{tabular}{|c|c|c|c|c|c|c|}
\hline Group & $\mathrm{N}$ & Age & \multicolumn{2}{|c|}{$\begin{array}{c}\text { Male } \\
\text { Female }\end{array}$} & Height (cm) & Mass (kg) \\
\hline $\begin{array}{l}\text { FOOT-TOE } \\
\text { ORTHOSIS }\end{array}$ & 24 & $21.83 \pm 1.74$ & 12 & 12 & $171.24 \pm 6.61$ & $71.84 \pm 13.96$ \\
\hline $\begin{array}{l}\text { SHOE } \\
\text { ONLY }\end{array}$ & 21 & $21.19 \pm 1.44$ & 9 & 12 & $174.78 \pm 11.63$ & $77.50 \pm 19.82$ \\
\hline CONTROL & 18 & $21.72 \pm 1.13$ & 9 & 9 & $171.45 \pm 8.18$ & $72.32 \pm 11.91$ \\
\hline Total & 63 & $21.59 \pm 1.49$ & 30 & 33 & $172.48 \pm 8.99$ & $73.86 \pm 15.64$ \\
\hline
\end{tabular}

\section{Instrumentation}

To assess dynamic balance, the Y-Balance Test Kit ${ }^{\circledR}$ (ybalancetest.com, Evansville, IN) was used (Figure 1). The test kit is constructed of three polyvinylchloride (PVC) pipes that attach to a central stance platform. The pipes are oriented in anterior, posteromedial, and posterolateral reach directions. The PVC pipes are marked in 5millimeter increments for measurement. Three plastic targets (reach indicators) sit atop the PVC pipes, which allows for precision of measurement and standardization of reach height. ${ }^{15}$ Correct Toes ${ }^{\mathrm{TM}}$ (Northwest Foot and Ankle, Portland, OR) is a foot-toe orthosis made of medical grade silicon that fits between the phalanges of the feet (Figure 2). It was necessary that the foot-toe orthosis be able to fit within the participant's footwear and that the footwear had an accommodating toe-box so subjects were fitted for and 
provided a manufacturer recommended control shoe (Primal 2, Lems Shoes ${ }^{\mathrm{TM}}$, Farrell, PA, Figure 3). Additionally, a cloth tape measure was used for assessing limb length of the individuals. Finally, a plastic goniometer was used for measuring the hallux valgus angle of the feet.

Y Balance Kit

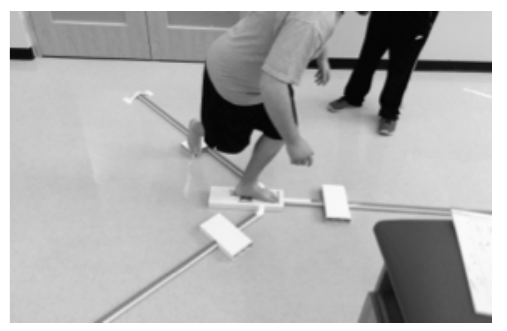

Figure 1.

\section{Correct Toes}

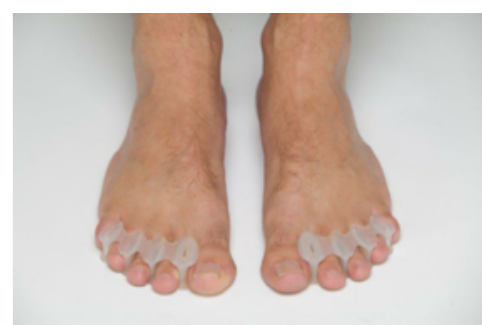

Figure 2.

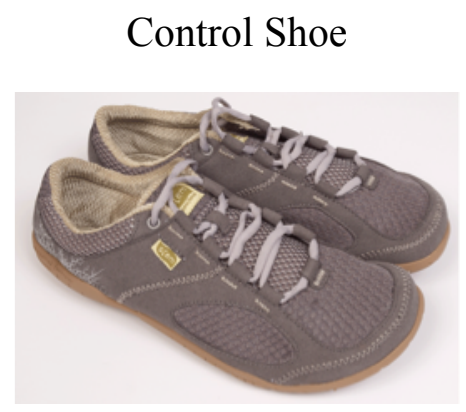

Figure 3. 


\section{Measurements}

Dynamic Balance was assessed using the Lower Quarter Y-Balance Test (YBTLQ) protocol, which is a validated and instrumented derivative of the SEBT. ${ }^{5,16,50}$ The tester used the Y-Balance Test Kit and the tester had completed the free online training (ybalancetest.com, Evansville, IN) for the YBT-LQ protocol and was certified to collect YBT-LQ data. Intra-rater reliability of the YBT-LQ was good $(\mathrm{ICC}=0.91, \mathrm{SEM}=1.46)$. Subjects were shown a short 2-minute tutorial video that explained the test and testing procedures as explained by Plisky et al. ${ }^{4}$ This video allowed for standardized instructions and increased familiarization with the testing procedures across all subjects. The YBTLQ assesses dynamic balance unilaterally and consists of reaching in three separate directions (anterior, posteromedial, and posterolateral) while maintaining unilateral stance on the contralateral limb. Subjects performed 6 practice trials in each reach direction on each leg prior to testing. These practice trials were performed to control for the learning effect associated with the testing protocol. ${ }^{16}$ A 2-minute rest period was provided before data collection began.

Subjects stood in single-leg stance barefoot on the center platform with the most distal aspect of the foot at the start line. Subjects were then asked to stand on one leg while reaching with the free limb in the anterior, posteromedial, and posterolateral directions. Each reach direction is labeled with reference to the stance leg. The established testing order used was three trials with the right leg reaching in the anterior direction followed by switching stance legs and then having the left leg reaching in the anterior direction. This order was repeated for the posteromedial and posterolateral reach directions. As part of the YBT-LQ protocol, a trial was discarded if the subject failed to 
maintain unilateral stance, touched down with the reaching foot, "kicked" or used excessive momentum to move the reach indicator, or failed to return to the starting position under control. The reach score was determined to the nearest half centimeter by the reach indicator's final resting location on the PVC pipe. The side of the reach indicator used for measurement was the side contacted by the most distal aspect of the reach foot. Three successful trials for each reach direction were recorded and the maximum reach score was used for data analysis. The aforementioned testing procedures were developed and established by Plisky et al. ${ }^{15}$ The composite score for the YBT-LQ was calculated by averaging the maximum reach scores for each direction after being normalized for limb length using the following formula. Composite Reach Score = $[($ Greatest Anterior Reach + Greatest Posteromedial Reach + Greatest Posterolateral Reach)/(3*Limb Length) $]^{*} 100$

To allow for normalization of reach distance, lower extremity limb length was assessed to the nearest half centimeter and was performed bilaterally for each subject. This was assessed with the subject in supine using a cloth tape measure to measure the distance from the inferior aspect of the anterior superior iliac spine to the most distal and inferior aspect of the medial malleolus. This was done after the examiner performed the Weber-Barstow maneuver ${ }^{51}$ on the subject to equalize the pelvis. The intra-rater reliability for measuring limb length was also high ( $\mathrm{ICC}=0.98, \mathrm{SEM}=0.89$ ).

For the purpose of this study, hallux valgus angle was defined as the angle formed by the intersection of the longitudinal axes of the proximal phalanx and the first metatarsal. ${ }^{52}$ A hallux valgus angle of less than 15 degrees is classified as normal. ${ }^{53}$ To assess the hallux valgus angle, the subject stood barefoot with feet shoulder width apart 
while the examiner palpated and then marked the skin at the location of the $1^{\text {st }}$ metatarsophalangeal joint, and also marked the longitudinal axis of the phalanges and $1^{\text {st }}$ metatarsal bone. These visual markings were then measured using a goniometer for an average of three trials to assess the subject's hallux valgus angle. The intra-rater reliability of the hallux valgus angle measurement was calculated to be ( $\mathrm{ICC}=0.93$, $\left.\mathrm{SEM}=1.6^{\circ}\right)$

\section{Procedures}

Upon arrival to the research laboratory, all participants were randomly allocated using the aforementioned method into either the Foot-Toe Orthosis and control shoe (FTO), shoe only (SO), or control group (CON) before undergoing baseline testing of dynamic balance and hallux valgus angle. Neither examiners, nor subjects were blinded to the subject's group. After undergoing baseline testing using the aforementioned procedures the FTO group was fitted for the foot-toe orthosis and the control shoe. There were two over-the-counter sizes (Small/Medium or Medium/Large) of the orthosis available for the participants. Sizing was determined by the examiner to ensure adequate fit and to avoid excessive splaying or discomfort. The control shoe size was provided based off the subject's normal footwear but adjustments in size were made to ensure adequate toe-box space for the orthosis.

The FTO group was instructed to wear the foot-toe orthosis in one of three ways: while barefoot, with or without socks, or while wearing the control shoe. Subjects were free to wear the foot-toe orthosis in any of these conditions as they chose and were able to switch between the options as needed for daily activities and comfort. They were also instructed not to wear the control shoe if they weren't concomitantly wearing the foot-toe 
orthosis. The FTO group wore the Correct Toes ${ }^{\circledR}$ for a four-week period that included gradual habituation before returning for post-testing of dynamic postural stability. The habituation period was implemented to prevent soreness or potential injury to the tissues of the feet and was based off the habituation period recommended by the product manufacturer. On the first day, the Correct Toes ${ }^{\circledR}$ were worn for approximately thirty minutes with an additional half-hour added per day afterwards. After completion of the habituation phase, the participants were instructed to wear the foot-toe orthosis for at least 4 hours a day and at night while sleeping. During the four week testing period, participants recorded information such as comfort of wear, and hours of wear on a daily basis in a $\log$.

The SO group was also fitted and provided the same control shoe as the FTO group but not the foot-toe orthosis. This group was also instructed to use the first week of the study as a habituation phase and then were instructed to wear the shoes for a minimum of 4 hours per day for the remaining 3-weeks of the study. Subjects in either group were allowed to wear the shoes and/or orthoses for more than 4 hours if they desired and granted they were not experiencing any pain or discomfort. It was also required that they record any additional wear time within their logs.

The CON group went about normal activities of daily living and all groups were instructed not to deviate from normal physical activity schedules for the duration of the study. All subjects then returned to the laboratory after four weeks to undergo follow-up testing. The follow-up testing utilized the same procedures as the baseline testing session. 


\section{Statistical Analysis}

An independent samples t-test was performed to determine whether leg dominance impacted performance on the YBT-LQ for the test subjects. A one-way between-groups analysis of covariance was conducted to compare the effectiveness of FTO and SO interventions to the CON group for improving dynamic balance. The independent variable was the type of intervention (FTO, SO, CON) and the dependent variable consisted of baseline and post-intervention YBT-LQ scores. Subject's baseline YBT-LQ scores were used as the covariate in this analysis. A one-way between groups analysis of covariance was also conducted to compare the effectiveness of the three interventions for influencing hallux valgus angle. The independent variable was the type of intervention (FTO, SO, CON) and the dependent variable consisted of baseline and post-intervention hallux valgus angles. Baseline hallux valgus angles were used as the covariate in this analysis. The hour logs were collected at follow-up and used to calculate means for hours of daily wear in each group to assess compliance.

An $\alpha$ level of $p \leq .05$ was set to determine statistical significance a priori. No correction for multiple comparisons was performed on the alpha level to protect against making a type II error. ${ }^{54}$ Instead, effect sizes (ES) were calculated based on the mean difference, the standard deviation (SD) of the differences, and the correlation of repeatedmeasures using Cohen's d with corresponding 95\% confidence intervals (CI). ES were interpreted as weak (0-0.39), moderate (0.40-0.69), and strong $(>0.70) .{ }^{55}$ All statistical analyses were performed using PASW (version 18.0, SPSS Inc., Chicago, IL). 


\section{CHAPTER IV}

\section{RESULTS}

The independent samples t-test found no significant difference $(p=.580)$ between dominant versus non-dominant limb performance on the YBT-LQ and so limbs were pooled together for each group prior to analysis by the ANCOVA. The mean daily average wear time for the FTO group was $4.03 \pm 1.21$ hours/day and was $3.81 \pm 0.91$ hours/day for the SO group. These were not significantly different $(p=.503)$ and all subjects were within \pm 1.5 standard deviations of the group mean and consequently no subjects were excluded as outliers.

Preliminary checks were conducted to ensure that there was no violation of the assumptions of normality, linearity, homogeneity of variances, homogeneity of regression slopes, and reliable measurement of the covariate. After adjusting for baseline scores, there were significant differences between the post-intervention scores on the YBT-LQ, $\mathrm{F}(2,126)=12.04, \mathrm{p}=.001$. Pairwise comparisons for revealed that the FTO group was significantly different $(\mathrm{p}=.001)$ from the CON group. There was a significant difference $(p=.034)$ between the SO and the CON groups. It was also revealed that the FTO group was significantly different ( $\mathrm{p}=.007)$ from the $\mathrm{SO}$ group. The FTO group $\mathrm{ES}=.70$ (0.291.12) was strong and the SO group $\mathrm{ES}=.45(0.01-0.88)$ was moderate. The ANCOVA revealed no significant differences $(\mathrm{p}=.380)$ in hallux valgus angle between any of the groups. 
Pre vs. Post YBT-LQ Composite Scores Chart

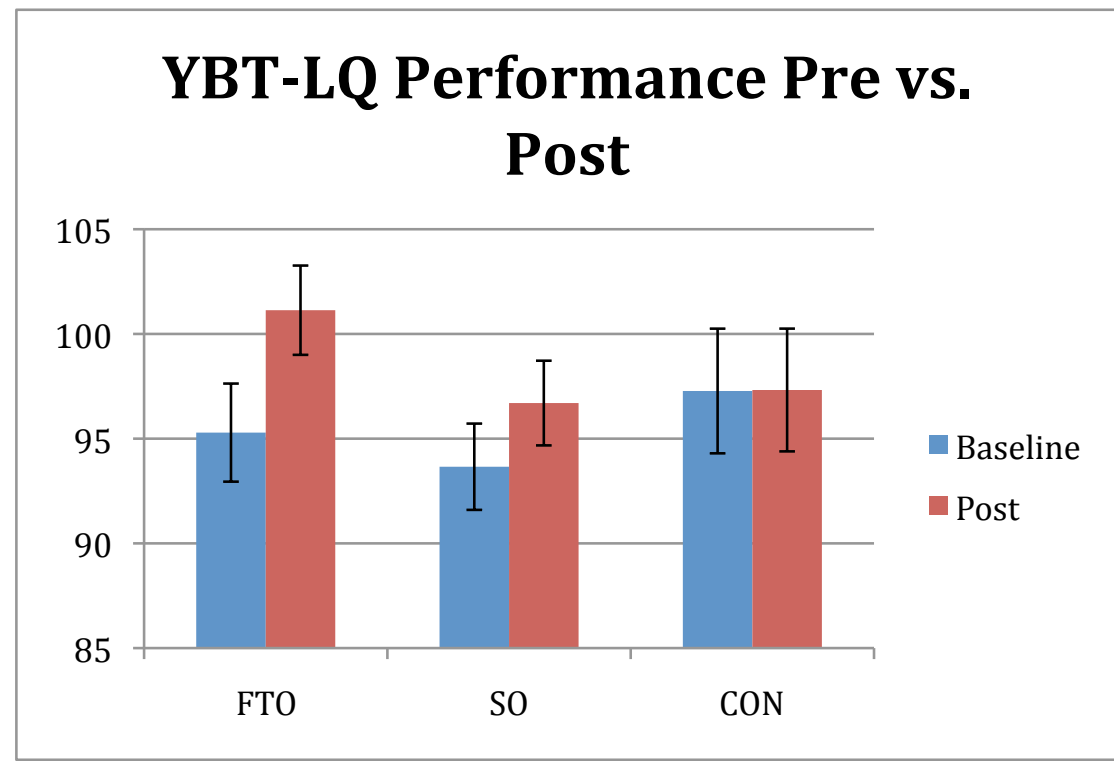

Figure 4

Table 2. Descriptive Statistics for YBT-LQ

\begin{tabular}{lccc}
\hline \multicolumn{1}{c}{ Group } & N & $\begin{array}{c}\text { Baseline Mean \& Std. } \\
\text { Deviation }\end{array}$ & $\begin{array}{c}\text { Post Mean \& Std. } \\
\text { Deviation }\end{array}$ \\
\hline FOOT-TOE ORTHOSIS & 48 & $95.30 \pm 8.29$ & $101.13 \pm 7.54^{* \wedge}$ \\
SHOE ONLY & 42 & $93.66 \pm 6.81$ & $96.70 \pm 6.69^{*}$ \\
CONTROL & 36 & $97.28 \pm 9.30$ & $97.32 \pm 8.97^{\wedge}$ \\
Total & 126 & $95.31 \pm 8.14$ & $98.57 \pm 7.92$ \\
\hline
\end{tabular}

* indicates a significant difference from the CON group

$\wedge$ indicates a significant difference from the SO group 


\section{CHAPTER V \\ DISCUSSION AND CONCLUSION}

\section{Discussion}

The purpose of this study was to investigate the effects of the foot-toe orthosis on dynamic stability and hallux valgus angle. Our results are the first to show the use of this foot-toe orthosis and/or the control shoe may cause an increase in dynamic balance over 4 weeks of use in a healthy young-adult population.

While our intervention was novel, our results were similar to other investigators that have used traditional orthotics, textured insoles, and textured surfaces to increase balance and stability. ${ }^{14,20,22,23,27,30,31,33-38}$ There are several proposed models of efficacy for using orthoses or insoles to improve balance. One theory is that orthoses may increase joint congruency via correcting any potential biomechanical mal-alignments. Another popular theory is that orthoses or insoles may potentially alter sensory afferent information derived from the plantar cutaneous surface and mechanoreceptors of the foot. ${ }^{20}$ Perry et al. ${ }^{31}$ found that textured insoles were able to increase postural stability in people with decreased foot-sole sensitivity. The increase in postural stability was still present after 12 weeks of continuous insole use by the participants. This finding supports the theory that postural stability requires constant input to the CNS, especially from the proprioceptive system. A lack of habituation to this input could represent a viable longterm method for increasing dynamic balance. 
The foot-toe orthosis used in this study was made of medical-grade silicon that is soft and flexible but provides a buffer that increases the joint space between the digits of the foot. Theoretically, the spacing provided by the Correct Toes ${ }^{\circledR}$ could have influenced the afferent feedback provided by the mechanoreceptors of the feet and toes to improve dynamic balance. In addition, the thin and flexible sole of the control shoe could have allowed increased afferent feedback as well. Furthermore, the increased inter-digital spacing provided by the foot-toe orthosis passively widened the base of support of the subject's feet while being worn. The wide-toe box design of the control shoe allowed for increased splaying of the feet; this theoretically could have resulted in an increased base of support as well during times of wear as well.

Our results showed the interventions for the FTO and SO groups had no effect on hallux valgus angle. These findings could lead to the assumption that the intervention's efficacy wasn't due to changes in anatomical alignment. However, the FTO group found significant improvements in dynamic balance after 4 weeks of use despite testing barefoot, and thus the improvements were not reliant upon constant afferent input or passive splaying of the toes from the foot-toe orthosis.

There are a couple of possible explanations for this inconclusive finding. One explanation is that the increased afferent input provided by the foot-toe orthosis altered the subject's motor control and movement patterns over time. The second is that the trial length (4 weeks) was an insufficient amount of time to allow for potential soft tissue adaptations or anatomical changes to take place. Another potential explanation is that the passive widening of the base of support provided by the foot-toe orthosis and control shoe during times of wear allowed for the changes seen in dynamic balance. 
Additionally, we failed to control for and measure other potential anatomical changes such as the width of the foot. This measure possibly could have been utilized to determine anatomical changes directly related to the subject's base of support. Another potential confounder was the method of measuring the hallux valgus angle. While pilot data calculated this method to have strong intra-rater reliability, we did not use the gold standard of radiographic measurements to assess the hallux valgus angle and thus this measure may not have been accurate enough. The long-term effects of the foot-toe orthosis and the control shoe on hallux valgus angle remain to be investigated.

This study only investigated the effects of the foot-toe orthosis and control shoe on dynamic balance and hallux valgus angle. Despite a theoretical model of influencing sensory-afferent input to the proprioceptive system or a potential for altering anatomical alignment this study did not investigate the veracity of these theoretical models. However, it is plausible that the efficacy of the interventions in this study is a combination of these theoretical models. Future research should be directed at investigating why this and other similar interventions are effective.

Some studies have had contradictory findings when attempting to use traditional foot orthoses or textured insoles to influence balance. ${ }^{23,25,27,56}$ However, direct comparison to these previous studies is difficult due to differences in our intervention, methodology, measures of balance or postural stability, and patient populations. We utilized a healthy patient population for FTO and SO groups in this study due to a lack of previous research. It would be beneficial for future and prospective research to investigate the effects of the foot-toe orthosis on injury rates, and dynamic balance in 
unhealthy, injured, or elderly populations. Elderly populations would specifically be of interest due to their high risk of falls.

The YBT-LQ has been identified as a sensitive tool for detecting subjects with underlying conditions such as ACL deficiency, chronic ankle instability, patellofemoral pain syndrome, and for evaluating the risk of injury in several populations. ${ }^{4,16-18}$ In a comparable population, de Noronha et al. ${ }^{18}$ found that healthy and physically active college students with decreased SEBT scores were at a higher risk for suffering lateral ankle sprains. It is warranted then that future research should investigate the effects of increasing dynamic balance with the foot-toe orthosis to potentially alter injury rates in similar populations.

There are a few potential limitations to our study. The tester and subjects were not blinded to the group allocation and this could have influenced motivation for testing performance. We attempted to counteract this by encouraging all subjects to give their best effort while testing and by providing standardized instructions to the subjects.

Secondly, our subjects were young men and women of a narrow age and physical activity range. Specifically, the majority of participants were undergraduate students with majors related to kinesiology and recreation. These subjects are probably more physically active and healthier than the average college student. This limits the ability for us to generalize our findings to other populations such as the elderly whom are at greater risk of falls. ${ }^{24}$ Another potential limitation to our study was the length of the trial. Four weeks is not a sufficient amount of time to see large adaptations in soft tissue.

Additionally, we do not know if habituation to the foot-toe orthosis and control shoe would occur after a longer period of use. Another limitation to this study is that we 
did not account or control for foot type in this study. We made this choice based on the

findings of a systematic review of the SEBT by Gribble and Plisky. ${ }^{16}$ Lastly, the foot-toe orthosis was only available in two separate sizes for our subjects. Considering the variety of foot size and structure for our subjects, we were sometimes left with a less than ideal fit. Fortunately, the manufacturer has added additional sizes and has special instructions for making modifications to the orthosis to accommodate differing foot types.

\section{Conclusion}

Our results suggest that the use of the foot-toe orthosis and the control shoe may increase dynamic balance in a healthy and college-aged population. The moderate to strong effect sizes associated with our results are promising; However, it is imperative that future research be conducted to investigate the effects in differing populations such as the elderly, the injured, and people with neuropathic conditions such as diabetes. It must also be investigated on whether these findings have any impact on injury risk and to determine what the long-term effects of use. This information could help researchers or clinicians investigate potential treatment or prophylactic approaches. 


\section{REFERENCES}

1. Hootman J. Epidemiology of collegiate injuries for 15 sports: Summary and recommendations for injury prevention initiatives. $J$ Athl Training. 2007;42(2):311.

2. Hertel J. Functional anatomy, pathomechanics, and pathophysiology of lateral ankle instability. J Athl Training. 2002;37(4):364.

3. Kannus P. Treatment for acute tears of the lateral ligaments of the ankle. operation, cast, or early controlled mobilization. J Bone Joint Surg. 1991;73(2):305.

4. Plisky PJ, Rauh MJ, Kaminski TW, Underwood FB. Star excursion balance test as a predictor of lower extremity injury in high school basketball players. J Orthop Sports Phys Ther. 2006;36(12):911-919.

5. Butler RJ, Southers C, Gorman PP, Kiesel KB, Plisky PJ. Differences in soccer players' dynamic balance across levels of competition. J Athl Train. 2012;47(6):616620.

6. Tropp H, Odenrick P. Postural control in single-limb stance. J Orthop Res. 1988;6(6):833-839.

7. Guskiewicz KM, Perrin DH. Research and clinical applications of assessing balance. $J$ Sport Rehabil. 1996(5):45-63.

8. Shumway-Cook A, Horak FB. Assessing the influence of sensory interaction of balance. suggestion from the field. Phys Ther. 1986;66(10):1548-1550.

9. Hazime FA, Allard P, Ide MR, Siqueira CM, Amorim CF, Tanaka C. Postural control under visual and proprioceptive perturbations during double and single limb stances: Insights for balance training. J Bodyw Mov Ther. 2012;16(2):224-229.

10. Tardieu C. Dental occlusion and postural control in adults. Neurosci Lett. 2009;450(2):221.

11. Gangloff P. Dental occlusion modifies gaze and posture stabilization in human subjects. Neurosci Lett. 2000;293(3):203.

12. Milani RS. Relationship between dental occlusion and posture. Cranio. 2000;18(2):127.

13. Nashner LM. Adaptation of human movement to altered environments. Trends Neurosci. 1982;5(0):358-361.

14. Corbin DM, Hart JM, McKeon PO, Ingersoll CD, Hertel J. The effect of textured insoles on postural control in double and single limb stance. J Sport Rehabil. 2007;16(4):363-372.

15. Plisky PJ, Gorman PP, Butler RJ, Kiesel KB, Underwood FB, Elkins B. The reliability of an instrumented device for measuring components of the star excursion balance test. N Am J Sports Phys Ther. 2009;4(2):92-99.

16. Gribble PA, Hertel J, Plisky P. Using the star excursion balance test to assess dynamic postural-control deficits and outcomes in lower extremity injury: A literature and systematic review. J Athl Train. 2012;47(3):339-357. 
17. Butler R. Dynamic balance performance and noncontact lower extremity injury in college football players: An initial study. Sports health. 2013;5(5):417.

18. de Noronha M. Intrinsic predictive factors for ankle sprain in active university students: A prospective study. Scand J Med Sci Sports. 2013;23(5):541.

19. Cobb SC, Tis LL, Johnson BF, Higbie EJ. The effect of forefoot varus on postural stability. J Orthop Sports Phys Ther. 2004;34(2):79-85.

20. Cobb SC, Tis LL, Johnson JT. The effect of 6 weeks of custom-molded foot orthosis intervention on postural stability in participants with $>$ or $=7$ degrees of forefoot varus. Clin J Sport Med. 2006;16(4):316-322.

21. Cote KP, Brunet ME, Gansneder BM, Shultz SJ. Effects of pronated and supinated foot postures on static and dynamic postural stability. $J$ Athl Train. 2005;40(1):41-46.

22. Gross MT, Mercer VS, Lin FC. Effects of foot orthoses on balance in older adults. $J$ Orthop Sports Phys Ther. 2012;42(7):649-657.

23. Guskiewicz KM, Perrin DH. Effect of orthotics on postural sway following inversion ankle sprain. J Orthop Sports Phys Ther. 1996;23(5):326-331.

24. Hatton AL, Dixon J, Rome K, Newton JL, Martin DJ. Altering gait by way of stimulation of the plantar surface of the foot: The immediate effect of wearing textured insoles in older fallers. J Foot Ankle Res. 2012;5:11-1146-5-11.

25. Hertel J, Denegar CR, Buckley WE, Sharkey NA, Stokes WL. Effect of rearfoot orthotics on postural sway after lateral ankle sprain. Arch Phys Med Rehabil. 2001;82(7):1000-1003.

26. Mattacola CG, Dwyer MK, Miller AK, Uhl TL, McCrory JL, Malone TR. Effect of orthoses on postural stability in asymptomatic subjects with rearfoot malalignment during a 6-week acclimation period. Arch Phys Med Rehabil. 2007;88(5):653-660.

27. McKeon PO, Stein AJ, Ingersoll CD, Hertel J. Altered plantar-receptor stimulation impairs postural control in those with chronic ankle instability. $J$ Sport Rehabil. 2012;21(1):1-6.

28. Ochsendorf DT, Mattacola CG, Arnold BL. Effect of orthotics on postural sway after fatigue of the plantar flexors and dorsiflexors. J Athl Train. 2000;35(1):26-30.

29. Olmsted L. Influence of foot type and orthotics on static and dynamic postural control. J Sport Rehab. 2004;13(1):54.

30. Palluel E, Olivier I, Nougier V. The lasting effects of spike insoles on postural control in the elderly. Behav Neurosci. 2009;123(5):1141-1147.

31. Perry SD, Radtke A, McIlroy WE, Fernie GR, Maki BE. Efficacy and effectiveness of a balance-enhancing insole. J Gerontol A Biol Sci Med Sci. 2008;63(6):595-602.

32. Sesma AR, Mattacola CG, Uhl TL, Nitz AJ, McKeon PO. Effect of foot orthotics on single- and double-limb dynamic balance tasks in patients with chronic ankle instability. Foot Ankle Spec. 2008;1(6):330-337.

33. Hartmann A, Murer K, de Bie RA, de Bruin ED. The effect of a training program combined with augmented afferent feedback from the feet using shoe insoles on gait performance and muscle power in older adults: A randomised controlled trial. Disabil Rehabil. 2010;32(9):755-764.

34. Witana CP, Goonetilleke RS, Xiong S, Au EY. Effects of surface characteristics on the plantar shape of feet and subjects' perceived sensations. Appl Ergon. 2009;40(2):267-279. 
35. Hatton AL, Dixon J, Rome K, Martin D. Standing on textured surfaces: Effects on standing balance in healthy older adults. Age Ageing. 2011;40(3):363-368.

36. Qiu F, Cole MH, Davids KW, et al. Enhanced somatosensory information decreases postural sway in older people. Gait Posture. 2012;35(4):630-635.

37. Hatton AL, Dixon J, Martin D, Rome K. The effect of textured surfaces on postural stability and lower limb muscle activity. J Electromyogr Kinesiol. 2009;19(5):957964.

38. Hamlyn C, Docherty CL, Klossner J. Orthotic intervention and postural stability in participants with functional ankle instability after an accommodation period. $J$ Athl Train. 2012;47(2):130-135.

39. Saladin KS. Anatomy \& physiology: The unity of form and function. 4th ed. New York, NY: McGraw-Hill; 2007:241-405.

40. Anderson MK, Parr GP, Hall SJ. Foundations of athletic training: Prevention, assessment, and management. In: 4th ed. Baltimore, Maryland: Lippincott Williams \& Wilkins, a Wolters Kluwer business; 2009:640-694.

41. Jansen EC, Larsen RE, Olesen MB. Quantitative romberg's test. measurement and computer calculation of postural stability. Acta Neurol Scand. 1982;66(1):93-99.

42. Sugano H, Takeya T. Measurement of body movement and its clinical application. The Japanese Journal of Physiology. 1970;20(3):296-308.

43. DIETZ V. Significance of proprioceptive mechanisms in the regulation of stance. Prog Brain Res. 1989;80:419.

44. G.A. Horstmann. The contribution of vestibular input to the stabilization of human posture: A new experimental approach. Neurosci Lett. 1988;95(1):179.

45. Meyer PF, Oddsson LI, De Luca CJ. The role of plantar cutaneous sensation in unperturbed stance. Exp Brain Res. 2004;156(4):505-512.

46. Kavounoudias A, Roll R, Roll JP. The plantar sole is a 'dynamometric map' for human balance control. Neuroreport. 1998;9(14):3247-3252.

47. Hertel J, Gay MR, Denegar CR. Differences in postural control during single-leg stance among healthy individuals with different foot types. J Athl Train. 2002;37(2):129-132.

48. Hoffman M, Payne VG. The effects of proprioceptive ankle disk training on healthy subjects. J Orthop Sports Phys Ther. 1995;21(2):90-93.

49. Matsusaka N, Yokoyama S, Tsurusaki T, Inokuchi S, Okita M. Effect of ankle disk training combined with tactile stimulation to the leg and foot on functional instability of the ankle. Am J Sports Med. 2001;29(1):25-30.

50. Hertel J. Simplifying the star excursion balance test: Analyses of subjects with and without chronic ankle instability. J Orthop Sports Phys Ther. 2006;36(3):131.

51. Seidenberg, Peter H. Bowen,Jimmy D. The hip and pelvis in sports medicine and primary care. [electronic resource]. Hip and Pelvis in Sports Medicine and Primary Care. 2010.

52. Coughlin MJ. Hallux valgus. J Bone Joint Surg Am. 1996;78(6):932-966.

53. HARDY RH. Observations on hallux valgus; based on a controlled series. The Journal of Bone and Joint Surgery.British Volume. 1951;33-B(3):376.

54. Perneger TV. What's wrong with bonferroni adjustments. BMJ.British medical journal. 1998;316(7139):1236-1238. 
55. Cohen J. Statistical power analysis for the behavioral sciences. Statistical Power Analysis for the Behavioral Sciences. 1988.

56. Percy ML. Effects of prefabricated foot orthoses and soft insoles on postural stability in professional soccer players. J Am Podiatr Med Assoc. 2001;91(4):194. 